Intersections

Canadian Journal of Music

Revue canadienne de musique
Intersections

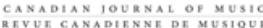

\title{
La musicologie rend hommage à Claude Lévi-Strauss
}

\section{Sophie Stévance}

Volume 30, numéro 1, 2010

URI : https://id.erudit.org/iderudit/1003495ar

DOI : https://doi.org/10.7202/1003495ar

Aller au sommaire du numéro

Éditeur(s)

Canadian University Music Society / Société de musique des universités canadiennes

ISSN

1911-0146 (imprimé)

1918-512X (numérique)

Découvrir la revue

Citer ce document

Stévance, S. (2010). La musicologie rend hommage à Claude Lévi-Strauss.

Intersections, 30(1), 3-4. https://doi.org/10.7202/1003495ar

All Rights Reserved (C Canadian University Music Society / Société de musique des universités canadiennes, 2011
Ce document est protégé par la loi sur le droit d'auteur. L'utilisation des services d’Érudit (y compris la reproduction) est assujettie à sa politique d'utilisation que vous pouvez consulter en ligne.

https://apropos.erudit.org/fr/usagers/politique-dutilisation/ 


\title{
ÉDITORIAL : LA MUSICOLOGIE REND HOMMAGE À CLAUDE LÉVI-STRAUSS
}

\author{
Sophie Stévance
}

Le 13 février 2009 se tenait à la Faculté de musique de l'Université de Montréal une journée d'étude consacrée à "Claude Lévi-Strauss : Hier et aujourd'hui ». L'événement était organisé par Nathalie Fernando, professeur agrégé occupant la chaire de recherche du Canada en ethnomusicologie, en collaboration avec le professeur Jean-Jacques Nattiez et le soutien de l'Observatoire interdisciplinaire de création et de recherche en musique (OICRM), ainsi que du Laboratoire de recherche sur les musiques du monde (LRMM). Il s'agissait de revenir sur l'apport ô combien considérable de Claude Lévi-Strauss quant à la compréhension du phénomène musical, mais aussi sur le rôle joué par la musique dans le développement des méthodes du fondateur du structuralisme en anthropologie (on relira à cet effet Le cru et le cuit, de 1964, ou encore L'Homme nu de 1971). Quelques ouvrages essentiels témoignent des rencontres de Lévi-Strauss avec la musique : que l'on songe notamment à l'impressionnant Lévi-Strauss musicien de Jean-Jacques Nattiez (Actes Sud, 2008) qui, sans vouloir diminuer le mérite des travaux traitant du même sujet, parle pour tous les autres.

À l'image de la thématique abordée au cours de cette importante journée d'études, le présent numéro d'Intersections ne démérite pas en prolongeant et surtout en donnant l'occasion d'approfondir - les réflexions des chercheurs réunis autour de l'événement du professeur Fernando. C'est ainsi que, dans "Lévi-Strauss - Brèves réflexions sur ses inspirations, sa démarche et son legs ", Nathalie Fernando introduit de manière convaincante à une pensée dense et féconde, mais aussi à l'homme dans son habit d'ethnologue engagé au cœur des sciences humaines, et également sujet à une réception ardente, souvent mitigée, mais toujours très stimulante. L'auteur démêle ainsi les nœuds avec doigté et livre une étude scientifique de haute tenue et d'une clarté irréprochable.

Ce souci d'éclairer le parcours intellectuel de ce grand voyageur est également le moteur liminaire du texte de Jean-Jacques Nattiez intitulé «La pensée de Lévi-Strauss comme objet esthétique». La plume vive et expressive de l'auteur, passionné par le débat des idées, érige les analyses de mythes de Lévi-Strauss en des catégories esthétiques générales - une finesse du regard qui aura tôt fait de retenir l'attention et le souffle du lecteur!

Ce beau témoignage relatant les rapports de Lévi-Strauss et de la musique, à plus forte raison l'admiration de Lévi-Strauss pour Wagner, est suivi de celui de "L'expérience sensible et son devenir abstrait. Comment deux amis ont lié d'amitié deux disciplines ». Signé par Nicole Revel, chercheur au Centre National de la recherche scientifique (CNRS, Laboratoire Langues-Musiques-Sociétés, Paris), 
cet article, dont la taille est amplement justifiée par la rigueur de l'argumentaire, se fait fort de mettre en lumière les liens étroits qui unissent la linguistique et l'anthropologie, et l'amitié entre Claude Lévi-Strauss et Roman Jakobson.

L'étude qui suit est celle de Monique Desroches, professeur titulaire d'ethnomusicologie à l'Université de Montréal. "Lévi-Strauss : homme de sens et de paradoxe» est un article approfondi dans lequel est examinée la conception d'un ethnologue (non de terrains mais féru de pensée humaine); l'auteur y propose des pistes de réflexion particulièrement intéressantes à partir de questionnements des positions méthodologiques et ethnographiques de Lévi-Strauss. Sous un œil attentif et aguerri, l'ethnomusicologue relève ainsi, sans pour autant passer sous silence la rigueur et l'originalité de ses travaux souvent pionniers, les aspects de la pensée de Lévi-Strauss faisant polémique.

C'est à ce titre que l'article du professeur Jonathan Goldman, intitulé «Structuralists contra Serialists? Claude Lévi-Strauss and Pierre Boulez on avant-garde music», revient sur les fondements d'un rendez-vous manqué entre les musiciens concrets, les sérialistes de la première heure, et Lévi-Strauss lui-même, que les uns ont fini par contester pour les propos sévères du dernier dirigés à l'encontre de la musique sérielle et concrète - mots publiés dès les premières pages de son important livre Le cru et le cuit, que le Lévi-Strauss musicien de Jean-Jacques Nattiez ou l'article de Nicolas Donin et de Frédéric Keck ("Lévi-Strauss et "la musique”. Dissonances dans le structuralisme», 2006) auront tôt fait d'analyser.

Mais au fil de la lecture de ces contributions, c'est bien la passion de LéviStrauss pour la musique qui retient toute notre attention. Le numéro s'achève ainsi sur "Mytheme and Motif. Lévi-Strauss and Wagner», du professeur John Leavitt, ethnolinguiste au département d'anthropologie de l'Université de Montréal. Dans cet article, l'auteur compare les «mythèmes» (découpage du récit en unités narratives) de Lévi-Strauss aux motifs répétés et transformés, fonctionnant comme éléments déclencheurs de rappel et de réponse, dans l'œuvre de Wagner. Bien que différents en soi, ces deux concepts ont pourtant de commun de produire sur l'auditeur un contentement tout à la fois sensible et intelligible, ce qui expliquerait la pérennité des mythes.

Toute l'équipe d'Intersections se joint à moi pour remercier chaleureusement les auteurs, plus particulièrement Nathalie Fernando et Jean-Jacques Nattiez qui nous ont fait confiance tout au long du processus de publication de ces "Actes» de l'événement qu'ils avaient organisé. Que ce témoignage de confiance marque également la poursuite de nos heureuses collaborations entre scientifiques francophones et anglophones, qui font la richesse de la recherche en musique au Canada, et dont Intersections veut se faire l'écho. Cette diversité est peut-être aussi (osons un tel rapprochement) à l'image de la pensée de ce grand voyageur et conteur à laquelle la grande famille de la musicologie rend aujourd'hui hommage : Claude Lévi-Strauss (1908-2009) n'a-t-il pas permis de penser ensemble différentes cultures, de pénétrer cette diversité essentielle à l'humanité pour ainsi bannir de son système dualiste, mais non moins dialogique, toute tentation d'ethnocentrisme? 